\title{
Compact amorphous-silicon visible-light monitor integrated in silicon nitride waveguides
}

\author{
Christian De Vita ${ }^{1}$, Charalambos Klitis ${ }^{2}$, Nina Codreanu ${ }^{1,3}$,Giorgio Ferrari ${ }^{1}$, Marc Sorel ${ }^{2}$, Andrea Melloni ${ }^{1}$, Francesco Morichetti ${ }^{1}$ \\ ${ }^{1}$ Department of electronics, information and bioengineering (DEIB), Politecnico di Milano, 20133 Italy \\ ${ }^{2}$ University of Glasgow, Rankine Building, Oakfield Avenue, Glasgow G12 8LT, UK \\ ${ }^{3}$ Now with: QuTech/Department of Quantum Nanoscience and Kavli Institute of Nanoscience, Delft University of Technology, Delft, The \\ Netherlands \\ christian.devita@polimi.it
}

\begin{abstract}
This work reports on the realization of an amorphous silicon visible-light detector integrated in $\mathrm{Si}_{3} \mathrm{~N}_{4}$ waveguides. The device is very compact $(<40 \mu \mathrm{m})$, has a responsivity of about 10 $\mathrm{mA} / \mathrm{W}$ and a sensitivity of $-40 \mathrm{dBm}$.
\end{abstract}

Keywords-optical waveguides, amorphous silicon, integrated detectors, visible light, photonic integrated circuits

\section{INTRODUCTION}

Visible-light is a spectral region of much interest for many applications such as imaging, metrology, biosensing, nanomedicine and quantum optics. In this wavelength range, Silicon Nitride ( $\mathrm{SiN}$ ) is one of the most established high-indexcontrast photonic platforms, offering a good tradeoff between low propagation loss and high integration scale [1]. In order to make photonic integrated circuits evolve towards more complex programmable architectures [2], it is necessary to monitor the working point of the photonic device by means of on-chip in-line light detectors.

Amorphous silicon (a-Si) is widely used in the visible range for the realization of opto-electronic devices, such as in photovoltaic applications. In integrated optics, a-Si is employed to realize optical waveguides for the near-infrared range where it is almost transparent, but in the visible range it is still largely unexploited. The integration of an a-Si detector on a lithium niobate waveguide on silicon has been recently demonstrated at $850 \mathrm{~nm}$ wavelength [3]. In this work, we exploit the photoconductivity of an a-Si film deposited as a coating layer of a SiN waveguide to realize a compact in-light photodetector operating in the red-light range. A sensitivity down to $-40 \mathrm{dBm}$ with a responsivity of $10 \mathrm{~mA} / \mathrm{W}$ is demonstrated.

\section{ASSESSMENT OF A-SI PHOTOCONDUCTIVITY}

The a-Si layer was deposited with a Silane based PECVD deposition: the presence of $\mathrm{SiH}_{4}$ will ensure the hydrogenation of the a-Si film, at the base of the photoconductive properties of amorphous the Silicon films [4]. An optimization process was performed in order to maximize the absorption coefficient of the a-Si film in the entire visible range of wavelengths in order to achieve the highest photoconductive response of the material. Material deposition was carried out with an RF power of $50 \mathrm{~W}$ at $13.56 \mathrm{MHz}$, a chamber pressure of $500 \mathrm{mTorr}$ and a substrate temperature of $300^{\circ} \mathrm{C}$.

The photoconductivity of the a-Si thin film at a wavelength $\lambda=660 \mathrm{~nm}$ was assessed by means of the vertically-illuminated test device shown in Fig 1(a). The $\mathrm{Au} / \mathrm{Cr}$ electrodes were realized through a lift-off process, by using direct laser writing lithography followed by a thermal evaporation process. As shown in Fig. 1(b), the light beam is vertically shone on the photoconductive film from a single-mode optical fiber, while two metallic probes are used to feed the device electrodes with the bias voltage and to read the light-dependent current through a lock-in read-out scheme. The I-V characteristics was firstly measured in DC in dark condition, this measurement providing a dark conductivity of the a-Si film of about $10^{-7} \mathrm{~S} / \mathrm{cm}$. Upon illumination see [Fig. 1(c)], we observed a dynamic range in the measured power intensity higher than $40 \mathrm{~dB}$ (from $-50 \mathrm{dBm}$ to $10 \mathrm{dBm}$ ), this change being responsible for a variation of more than three orders of magnitude in the a-Si conductivity (quantified in about $10^{-4} \mathrm{~S} / \mathrm{cm}$ for $-9 \mathrm{dBm}$ input power). The slight sublinearity in the current-power characteristic is due to the reduction of the free-carrier lifetime in the a-Si film at high carrier generation rate, that is at higher photon flux) [4].

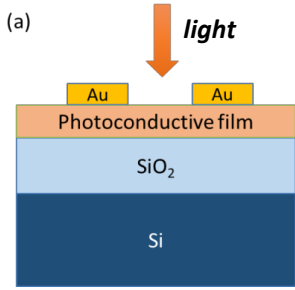

(b)

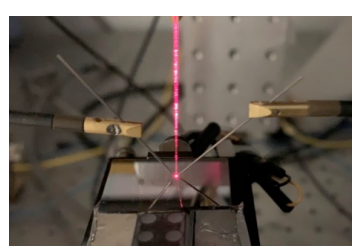

(c)

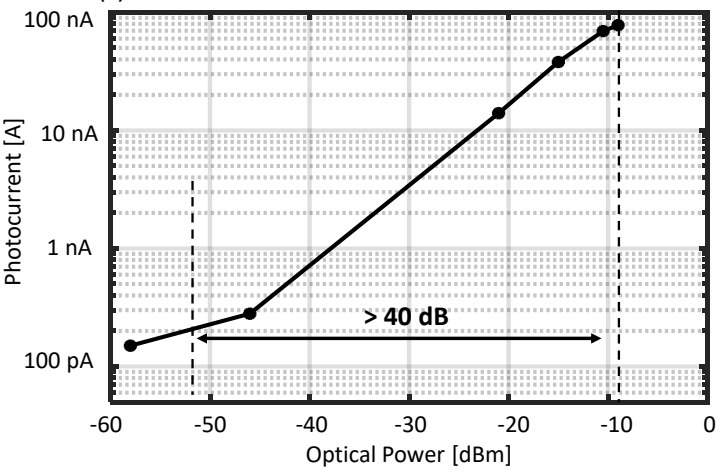

Figure 1. (a) Schematic of the vertically-illuminated device for the assessment of a-Si photoconductivity. (b) Photograph of the experimental setup. c) Photocurrent measured in vertically-illuminated test devices (electrode size $200 \mu \mathrm{m}$ x $200 \mu \mathrm{m}$, electrode spacing 20 $\mu \mathrm{m}$ ) for increasing optical power at a wavelength of $660 \mathrm{~nm}$ (red light). 
This effect is responsible for a reduction of the responsivity at higher optical power; however, at maximum optical power of $-9 \mathrm{dBm}$, a photocurrent of $80 \mathrm{nA}$ is measured $(20 \mu$ m electrode spacing, $8 \mathrm{~V}$ applied voltage) resulting in a responsivity of about $5 \mathrm{~mA} / \mathrm{W}$, which is close to recently reported results for a-Si photoconductors [3].

\section{A-Si DETECTOR INTEGRATED IN $\mathrm{SI}_{3} \mathrm{~N}_{4}$ WAVEGUIDES}

Figure 2(a) schematically shows the longitudinal geometry of the a-Si detector integrated in $\mathrm{Si}_{3} \mathrm{~N}_{4}$ waveguide. The hydrogen silsesquioxane (HSQ) upper cladding of a buried channel waveguide $\left(\mathrm{Si}_{3} \mathrm{~N}_{4}\right.$ core $400 \mathrm{~nm} \times 200 \mathrm{~nm}$ ) is locally lowered in order to enable the deposition of a thin film of a-Si at a suitable spacing from the waveguide in order to introduce a controllable absorption of the guided light. Electromagnetic simulations based on the beam propagation method (BPM) show that the evanescent coupling between the fundamental transverse electric (TE) mode and a $200 \mathrm{~nm}$ thick a-Si film coating layer at a distance of $200 \mathrm{~nm}$ provides $4 \mathrm{~dB}$ absorption over $100 \mu \mathrm{m}$ propagation length. Figure 2(b) shows a top view microphotograph of a fabricated device. Selective removal of the HSQ upper cladding was performed by direct laser writing optical lithography followed by reactive ion etching in $\mathrm{CHF}_{3} / \mathrm{O}_{2}$ mixture. Once deposited, the a-Si is then patterned by direct laser writing optical lithography and then dry etched in $\mathrm{SF}_{6} / \mathrm{O}_{2}$ mixture. On top of the a-Si film a pair of electric contact pads (4 $\mu \mathrm{m}$ spacing, $15 \mu \mathrm{m}$ width) were fabricated by lift-off technique using a direct laser writing optical lithography followed by the deposition of $150 \mathrm{~nm}$ of gold with a small adhesion layer of chromium. The bonded pads are kept as far as possible in order to avoid unwanted capacitances, while the two golden paths are kept very close in order to minimize the resistive path of the current in the a-Si.

The laser source is butt-coupled to the waveguide device by using a horizontal alignment optical setup. The optical fiber has a mode field diameter of about $3.5 \mu \mathrm{m}(+/-0.5)$ providing a coupling loss of about $10 \mathrm{~dB} /$ facet with the optical waveguide if no mode adapters are realized at the waveguide termination. In order to reduce the parasitic capacitances due to the electrical probes, the sample was wire bonded to an electrical. Figure 2(c) shows the measured photocurrent versus the frequency of the lock-in read-out electronics for different optical power in the detector region. The plateau in the low-frequency side of the curves provides the light dependent change of the photocurrent due to the photoconductivity of the a-Si film, whereas the high frequency behavior is dominated by the parasitic capacitance between the metallic pads. The black line provides a reference dark current of $65 \mathrm{pA}$ (at $8 \mathrm{~V}$ applied voltage). When the light beam is switched on, a photocurrent of about $4 \mathrm{nA}$ is measured (red line), with an estimated power at the sensor of $-30 \mathrm{dBm}$. and an absorption of $2 \mathrm{~dB}$. This photocurrent corresponds to a responsivity of about $10 \mathrm{~mA} / \mathrm{W}$, that is similar to the one we obtained for the vertically illuminated device. The maximum frequency of the detector is currently set by the parasitic capacitance limiting the operational bandwidth to $1 \mathrm{kHz}$ at -30 $\mathrm{dBm}$, which is however in line with typical monitoring operation but that can be used down to almost $-50 \mathrm{dBm}$ with longer reading time
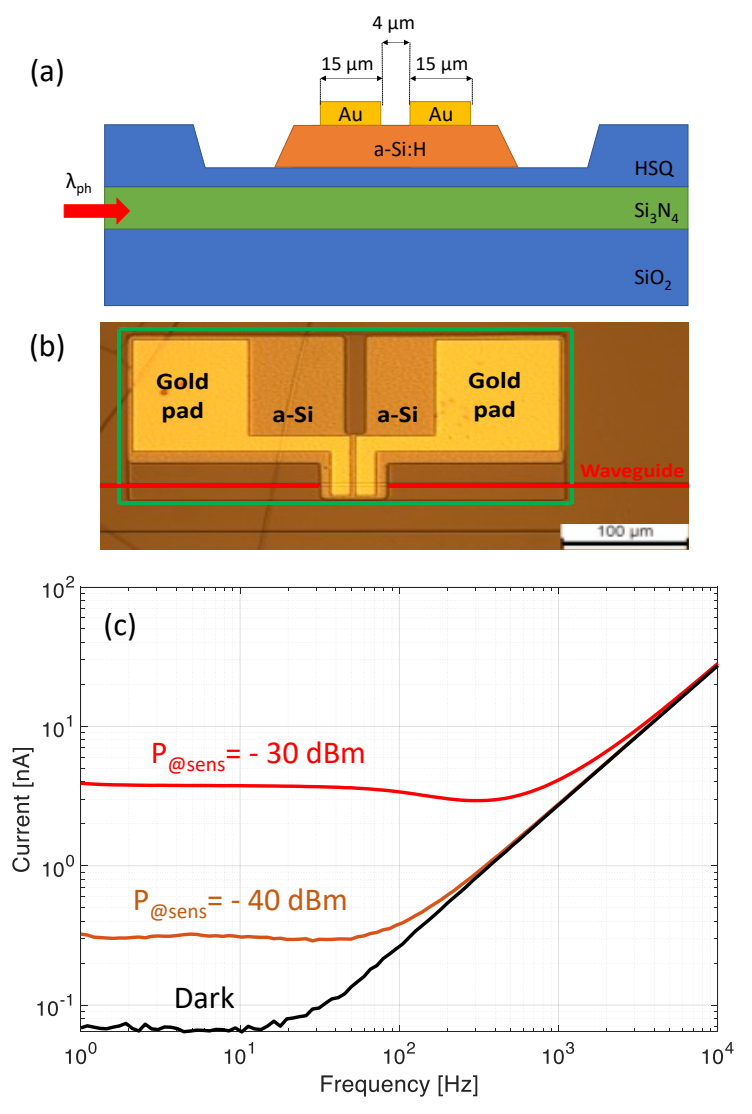

Figure 2. (a) Schematic of the a-Si detector integrated in a $\mathrm{Si}_{3} \mathrm{~N}_{4}$ waveguide; (b) Top view photograph of a fabricated device; (c) Measured photocurrent versus the frequency of the electrical lock-in read-out for increasing optical power at the detector: dark condition (black curve), $-40 \mathrm{dBm}$ (brown), and - $30 \mathrm{dBm}$ (red curve).

\section{ACKNOWLEDGMENT}

We acknowledge Stefania Intelisano and the technical staff of the JWNC at Glasgow University and of Polifab, the micro- and nanofabrication facility of Politecnico di Milano, for support in the fabrication of the devices. This supported by European Commission through H2020 grant number 829116 (SuperPixels).

\section{REFERENCES}

[1] Wesley D. Sacher et al. "Visible-light silicon nitride waveguide devices and implantable neurophotonic probes on thinned $200 \mathrm{~mm}$ silicon wafers," Opt. Express 27, 37400-37418 (2019)

[2] Bogaerts, W., Pérez, D., Capmany, J. et al. Programmable photonic circuits. Nature 586, 207-216 (2020). https://doi.org/10.1038/s41586020-2764-0

[3] B. Desiatov and M. Loncar. "Silicon photodetector for integrated lithium niobate photonics". Appl. Phys. Lett. 115, 121108 (2019).

[4] C. R. Wronski and R. E. Daniel, "Photoconductivity, trapping, and recombination in discharge-produced, hydrogenated amorphous silicon," Phys. Rev. B 23, 794 (1981). 